\title{
SEMI GENERALIZED OPEN SETS AND GENERALIZED SEMI CLOSED SETS IN TOPOLOGICAL SPACES
}

\author{
ABDELGABAR ADAM HASSAN1,2,* \\ ${ }^{1}$ Department of Mathematics, College of Science and Arts in Tabrjal, Jouf University, Kingdom of Saudi \\ Arabia \\ 2Department of Mathematics, University of Nyala, Nyala, Sudan \\ *Corresponding author: aahassan@ju.edu.sa
}

ABSTRACT. In this paper we introduce a new class of semi generalized open sets, generalized semi closed sets in topological spaces, and studied some of its basic properties. Moreover we define approximately semi generalized open sets and approximately generalized semi closed sets in topological spaces. Further we obtained some properties of closure, semi generalized open sets and generalized semi closed sets in topological spaces.

\section{INTRODUCTION}

The study of generalized closed sets in topological space was initiated by Levine in [23]. Biswas [18], Njasted[15], Mashhour[12], Robert[19], Bhattacharya [13], Arya and Nour [12 ]. introduced and investigated semi closed, -open and -closed, pre-open, semi*-open, sg-closed, gsclosed, gp-closed, g-closed, $\mathrm{g}^{*}$ closed, $\mathrm{s}^{*} \mathrm{~g}$-closed, $\mathrm{w}$-closed, $\mathrm{g}^{*}$-closed respectively. Topology is an important and interesting area of mathematics, the study of which will not only introduce to new concepts and theorems but also put into context old ones like continuous functions [1]. However, to say just this is to understate the significance of topology. It is so fundamental that its influence is evident in almost every other branch of mathematics [3]. Topological notions like compactness, connectedness and denseness areas basic to mathematicians of today as sets and functions were

Received March 31st, 2020; accepted June 1st, 2020; published October 13"th, 2020.

2010 Mathematics Subject Classification. 54A05.

Key words and phrases. topological spaces; semi generalized open sets; semi generalized closed sets.

(C)2020 Authors retain the copyrights of their papers, and all open access articles are distributed under the terms of the Creative Commons Attribution License. 
to those of last century. Topology has several different branches, genera 1 topology, algebraic topology, differential topology and topological algebra, the first, general topology, being the door to the study of the others $[3,5]$.

The aim of this paper is to introduce the concept of semi generalized open Sets and generalized semi closed Sets in topological spaces, and provide Semi generalized open Sets and generalized semi closed Sets in topological spaces.

\section{PRELIMINARIES}

Definition 2.1. Let $X$ be a non - empty sets. A collection of subsets of $X$ is said to be a topology on $X$ if

(i) $\quad X$ and the empty set, $\varnothing$, belong to $\tau$,

(ii) The union of any (finite or infinite) number of sets in $\tau$ belong to $\tau$,

(iii) The intersection of any two sets in $\tau$ belongs to $\tau$.

The pair $(X, \tau)$ is called a topological space.

Definition 2.2 Let $X$ be a non - empty sets and let $\tau$ be the collection of all subsets of $X$. Then $\tau$ is called discrete topology on the set $X$. The topological space $(X, \tau)$ is called a discrete space.

Observe that the set $X$ in Definition 1.2. can be any non - empty set. So, there is an infinite number of discrete spaces - one for each $X$.

Definition 2.3. Let $X$ be any non - empty set and $\tau=\{X, \emptyset\}$. Then $\tau$ is called indiscrete topology and $(X, \tau)$ is said to be indiscrete space.

Definition 2.4. Let $(X, \tau)$ be a topological space. A subset $A$ of $X$ is said to be generalized closed if $\operatorname{cl}(A) \subseteq U$ when ever $A \subseteq U$ and $U$ is an open in $(X, \tau)$.

Definition 2.5. Let $(X, \tau)$ be a topological space and $A \subseteq X$. The generalized closure of $A$, denote by $c l^{*}(A)$ and is defined by the intersection of all $g$ closed sets containing $A$ an generalized interior of $A$, denoted by $\operatorname{int}^{*}(A)$ and is defined by union of all $g$ - open sets contained in $A$.

Definition 2.6. A subset $A$ of a topological space $(X, \tau)$ is said to be a semi - open set [9] if $A \subseteq \operatorname{cl}(\operatorname{int}(A))$ and a semi closed if $\operatorname{int}(\operatorname{cl}(A)) \subseteq A$, a preopen set [11] if $A \subseteq \operatorname{int}(\operatorname{cl}(A))$ and a pre closed if $\operatorname{cl}(\operatorname{int}(A)) \subseteq A$, an $\propto$ - open sets [12] if $A \subseteq \operatorname{int}(\operatorname{cl}(\operatorname{int}(A)))$ and $\propto$ - closed sets if $\operatorname{cl}(\operatorname{int}(\operatorname{cl}(A))) \subseteq A$,

(iv) A regular set [13] if $\operatorname{int}(\operatorname{cl}(A))=A$ and a regular closed set if $\operatorname{cl}(\operatorname{int}(A))=A$, 
(v) A $Q-\operatorname{set}[10]$ if $\operatorname{cl}(\operatorname{int}(A))=\operatorname{int}(\operatorname{cl}(A))$.

The intersection of all semi - closed (resp. to pre closed, $\propto$ - closed) sets containing a subset $A$ of $(X, \tau)$ is called semi - closure [14] (resp. pre closure, $\propto$ - closed, $c l_{\alpha}(A)$. The semi - interior of $A$ is the largest semi - open set contained in $A$ and denoted by $S-\operatorname{int}(A)$.

\section{OPEN SETS AND CLOSED SETS IN TOPOLOGICAL}

Definition 3.1. Let $(X, \tau)$ be any topological space. Then the members of $\tau$ are said to be open sets.

Proposition 3.2. If $(X, \tau)$ is any topological space, then $X$ and $\emptyset$ are open sets, the union of any (finite or infinite) number of open sets is an open set, the intersection of any finite number of open sets is an open set.

Proof. Clearly (i) and (ii) are trivial consequences of Definition 2.1. and Definition 2.1.(i) and (ii). The condition (iii) follows from Definition 2.1.

Definition 3.3. Let $(X, \tau)$ be a topological space. A subset $S$ of $X$ is said to be a closed set in $(X, \tau)$ if its complement in $X$, namely $X \backslash S$, is open in $(X, \tau)$.

Proposition 3.4. If $(X, \tau)$ is any topological space, then $\emptyset$ and $X$ are closed sets, the intersection of any (finite or infinite) number of closed sets is a closed set, the union of any finite number of closed sets is a closed set.

Proof. (i) follows immediately Proposition 2.2. and Definition 2.3., as the complement of $X$ is $\emptyset$ and the complement of $\varnothing$ is $X$.

To prove that (iii) is true, let $S_{1}, S_{2}, \ldots, S_{n}$ be closed sets. We are required to prove that $S_{1} \cup S_{2} \cup$ $\ldots \cup S_{n}$ is a closed set. It suffices to show, by Definition 2.3., that $X \backslash\left(S_{1} \cup S_{2} \cup \ldots \cup S_{n}\right)$ is an open set.

As $S_{1}, S_{2}, \ldots, S_{n}$ are closed sets, their complement $X \backslash S_{1}, X \backslash S_{2}, \ldots, X \backslash S_{n}$ are open sets. But

$$
X \backslash\left(S_{1} \cup S_{2} \cup \ldots \cup S_{n}\right)=\left(X \backslash S_{1}\right) \cap\left(X \backslash S_{2}\right) \cap \ldots \cap\left(X \backslash S_{n}\right)
$$

As the right hand side of (1) is a finite intersection of open sets, it is an open set. So that the left hand side of (1) is an open set. Hence $S_{1} \cup S_{2} \cup \ldots \cup S_{n}$ is a closed set, as required. So (iii) is true. 
The proof of (ii) is similar to that of (iii).

Example 3.5. On any set $X$ there is the trivial topology $\{\varnothing, X\}$. There is also the discrete topology whereas any subset of $X$ is open. Thus, on a set there can be many topologies.

Example 3.6. (Euclidean topology). In $\mathbb{R}^{n}=\left\{\left(x_{1}, x_{2}, \ldots, x_{n}\right) \mid x_{i} \in \mathbb{R}\right\}$, the Euclidean norm of a point $x=\left(x_{1}, x_{2}, \ldots, x_{n}\right)$ is $\|x\|=\left[\sum_{i=1}^{n} x_{i}^{2}\right]^{1 / 2}$. The topology generated by this norm is called the Euclidean topology of $\mathbb{R}^{n}$.

\section{SEMI GENERALIZED * b OPEN SETS}

In this part, we introduce semi generalized* $b$ open sets in topological spaces by using the notion of semi generalized b- open sets, and study some of their properties.

Definition 4.1. A subset $A$ of a topological space $(X, \tau)$, is called semi generalized * $\mathrm{b}$ open set if $A^{c}$ is semi generalized - $\mathrm{b}$ closed in $X$.

We denote the family of all semi generalized - b open sets in $X$ by $s g b-O(X)$.

Theorem 4.2. If $A$ and $B$ are $s g$-open sets in a space $X$. Then $A \cap B$ is also $s g b$ - open set in $X$.

Proof. If $A$ and $B$ are $s g b$-open sets in a space $X$. Then $A^{c}$ and $B^{c}$ are $s g b$ - closed sets in a space $X$. Therefore $A^{c} \cup B^{c}$ is also $s g b$ - closed set in $X$. (i.e.) $A^{c} \cup B^{c}=(A \cap B)^{c}$ is a $s g b$ closed set in $X$. Therefore $A \cap B s g b$ - open set in $X$.

\section{SEMI GENERALIZED * $b$ CLOSED SETS}

In this part, we introduce semi generalized* $b$ - closed set and investigate some of its properties.

Definition 5.1. (Closed set). Let $(X, \tau)$ topological space. the set $A \subseteq X$ is called closed set if and only if $A^{C}$ be open set. i.e.

$A$ closed set $A \Leftrightarrow A^{C}$ open set

$A$ open set $A \Leftrightarrow A^{C}$ closed set

And denote that by $\mathfrak{I}_{X}$ or $\mathfrak{J}$.

Example 5.2. Let the set $X=\{a, b, c, d, e\}$. Define topology $\tau$ into $X$ by the flowing:

$$
\tau=\{X, \emptyset,\{a\},\{c, d\},\{a, c, d\},\{b, c, d, e\}\}
$$

Then

$$
\mathfrak{I}_{X}=\{X, \emptyset,\{b, c, d, e\},\{a, b, e\},\{b, e\},\{a\}\} .
$$


Example 5.3. Let $(X, D)$ Discrete topological space. We find that each subset from $(X, D)$ is open and closed at the same time because:

$$
P(X)=D, \forall A \subseteq X \Leftrightarrow A \in D \Leftrightarrow A^{C} \text { closed }
$$

Definition 5.4. A subset $A$ of a topological space $(X, \tau)$, is called semi generalized * $\mathrm{b}$ closed set if $\operatorname{cl}(A) \subset \mathrm{U}$ whenever $A \subset \mathrm{U}$ and $\mathrm{U}$ is semi generalized - b open in $X$.

Theorem 5.5. Every closed set is $s g b$ - closed.

Proof. Let $A$ be any closed set in $X$ such that $A \subset U$, where $U$ is $s g$ open. Since $b \operatorname{cl}(A) \subset \operatorname{cl}(A)=$ $A$. Therefore $b \operatorname{cl}(A) \subset \cup$. Hence $A$ is $s g b$ - closed set in $X$. Is

Example 5.6. Let $X=\{a, b, c\}$ with $\tau=\{X, \emptyset,\{b\},\{a, b\}\}$. The set $\{a, b\}$ is $s g b$ - closed set but not a closed set.

Theorem 5.7. Every semi closed set is $s g b$-closed set.

Proof. Let $A$ be any closed set in $X$ and $U$ be any $s g$ open set containing $A$. Since $A$ is semi closed set, $b \operatorname{cl}(A) \subset S \operatorname{cl}(A) \subset \cup$. Therefore $b c l(A) \subset U$. Hence $A$ is $s g b$ - closed set.

Corollary 5.8. Let $X=\left\{a_{1}, a_{2}, a_{3}\right\}$ with $\tau=\left\{X, \emptyset,\left\{a_{1}, a_{2}\right\}\right\}$. The set $\left\{a_{1}, a_{2}\right\}$ is $s g b$-closed set but not a semi closed set.

Theorem 5.9. Every $\propto$ - closed set is $s g b$ - closed set.

Proof. Let $A$ be any $\propto$-closed set in, and $X$ and $U$ be any $s g$ set containing $A$. Since $A$ is $\propto$-closed $b \operatorname{cl}(A) \subset \propto \operatorname{cl}(A) \subset \mathrm{U}$. Therefore $b c l(A) \subset \mathrm{U}$. Hence $A$ is $s g b$ - closed set.

Theorem 5.10. Every $s g b$-closed set is $g b$-closed set.

Proof. Let $A$ be any $s g b$-closed set in, such that $U$ be any open set containing $A$. Since every open set is $s g$ open, we have $b \operatorname{cl}(A)$.. Hence $A$ is $g b$ - closed set.

\section{THE CLOSURE AND INTERIOR}

Definition 6.1. Let $X$ be a topological space and $A \subseteq X$ a subset. The closure of $A$ denote $\bar{A}$ is the intersection of all the closed subsets of $X$ that contain $A$. The interior of $A$ is the union of all the open subsets of $X$ that are contained in A.

Example 6.2. Let $X=\mathbb{R}$ and $\mathrm{A}=[\mathrm{a}, \mathrm{b})$ with $\mathrm{a}<\mathrm{b}$. The closure of $A$ is the closed interval [a, $\mathrm{b}]$, and the interior of $A$ is the open interval $(\mathrm{a}, \mathrm{b})$. The closure cannot be smaller, since $[\mathrm{a}, \mathrm{b})$ is not closed, and the interior cannot be larger, since[a,b) is not open.

Theorem 6.3. Let $X$ be a topological space, $Y \subset X$ a subspace, and $A \subset Y$ a subset. Let $\bar{A}$ denote the closure of $A$ in $X$. Then the closure of $A$ in $Y$ equals $\mathrm{Y} \cap \overline{\mathrm{A}}$ 
Proof. Let $B$ denote the closure of $A$ in $Y$.

To see that $\mathrm{B} \subset \mathrm{Y} \cap \overline{\mathrm{A}}$, note that $\bar{A}$ is closed in $X$, so $\mathrm{Y} \cap \overline{\mathrm{A}}$ is closed in $Y$ and contains $A$. Hence it contains the closure $B$ of $\mathrm{A}$ in $Y$.

To prove the opposite inclusion, note that $B$ is closed in $Y$, hence has the form $\mathrm{B}=\mathrm{Y} \cap \mathrm{C}$ for some $\mathrm{C}$ that is closed in $X$. Then $\mathrm{A} \subset \mathrm{B} \subset \mathrm{C}$, so $\mathrm{C}$ is closed in $X$ and contains $A$. Hence $\overline{\mathrm{A}} \subset \mathrm{C}$ and $\mathrm{Y} \cap \overline{\mathrm{A}} \subset \mathrm{Y} \cap \mathrm{C}=\mathrm{B}$.

\section{Example 6.4. Topologist 's sine curve.}

The closure in the Euclidean plane of the graph of the function $y=\sin \frac{1}{x}, x>0$ is often called the Topologist's sine curve.

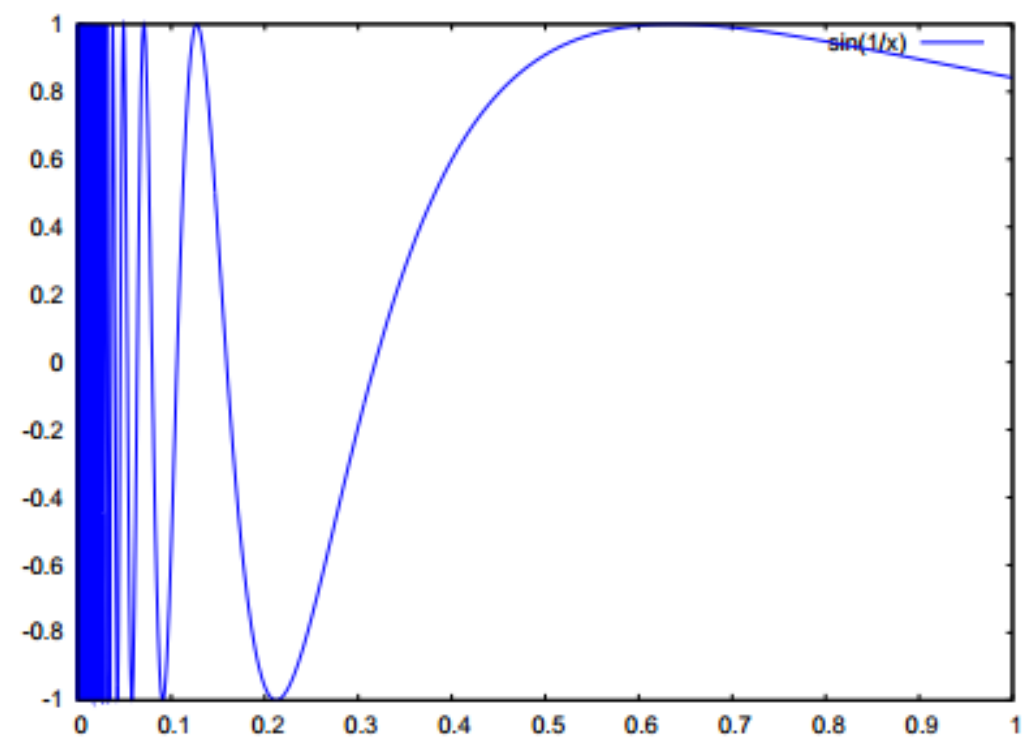

Figure 1: Topologist's sine curve.

Denote $A=\left\{\sin \frac{1}{x} \mid x>0\right\}$ and $B=\{0\} \times[-1,1]$. Then the Topologist's sine curve is $X=A \cup B$.

\section{CONCLUSION}

The aim of this paper is to introduce the concepts of semi generalized open Sets and generalized semi closed Sets in topological spaces sets and we study some of their properties. Furthermore, we discuss the conditions which are added to these concepts in order to coincide with the concept of semi-closed. 
Data Availability: No data were used to support this study.

Acknowledgment: I'm forever indebted my family for their endless patience and encouragement, also I want to recognize and express my thank to anyone helped me.

Conflicts of Interest: The author declares that there are no conflicts of interest regarding the publication of this paper.

\section{References}

[1] J. Dugundji, Topology, Allyn and Bacon, Inc., Boston. 1966.

[2] S.A. Morris, Topology without tears, Unisia, 2001.

[3] S. Sekar, B. Jothilakshmi, On semi generalized star b - closed set in topological spaces, Int. J. Pure Appl. Math. 113 (2017), 93-102.

[4] S. Lipschutz, Schaum's outline of theory and problems of general topology, McGraw-Hill, New York. 1965.

[5] D. Lyappan, N. Nagaveni, On semi generalized b-continuous maps, semi generalized b-closed maps in topological space, Int. J. Math. Anal. 6 (2012), 1251-1264.

[6] S. S. Benchalli, P. G. Patil, P. M. Nalwad, Generalized wa-Closed Sets in Topological Spaces, J. New Results Sci. 7 (2014), 7-19.

[7] N. Biswas, On Characterization of semi - continuous functions, Atti Accad. Naz. Lincei. Rend. Cl. Sci. Fsi. Mat. Natur. 48 (1970), 399-402.

[8] P. Bhattacharyya, B. K. Lahiri, Semi-generalized closed sets in topology, Ind. J. Math. 29 (1987), 375382.

[9] N. Biswas, On characterizations of semi-continuous functions, Atti. Accad. Naz. Lincei Rend. Cl. Sci. Fis. Mat. Natur. 8 (1970), 399-402.

[10]S.G. Crossly, S.K. Hildebrand, Semi- closure, Texas J. Sci. 22(1971), 99-112.

[11]S.G. Crossly, S.K. Hildebrand, Semi- topological properties, Fund. Math. 74 (1974), 233-254.

[12] S.P. Arya, T. Nour, Characterizations of S- normal spaces, Indian J. Pure. Appl. Math. 21 (8) (1990), 717719.

[13] P. Bhattacharya, B. K. Lahiri, Semi- generalized closed sets in Topology, Indian J. Math. 29 (1987), 376382.

[14]R. Devi, Studies on generalizations of closed maps and homeomorphisms in topological spaces, Ph.D. Thesis, Bharathiar University, Coimbatore (1994). 
[15] N. Levine, On the commutivity of the closure and interior operator in topological spaces, Amer. Math. Mon. 68 (1961), 474-477.

[16] N. Levine, Semi-open sets and semi-continuity in topological spaces, Amer Math. Mon. 70 (1963), 3641.

[17] N. Levine, Generalized closed sets in topology, Rend. Circ. Mat. Palermo, 19 (1970), 89-96.

[18] N. Biswas, On characterizations of semi- continuous functions, Atti. Accad. Naz. Lincei. Rend. Cl. Sci. Fis. Mat. Natur. 48 (1970), 399-402.

[19]S. S. Benchalli, P. G. Patil, T. D. Rayanagaudar, wa-Closed Sets is Topological Spaces, Glob. J. Appl. Math. Math. Sci. 2 (2009), 53-63.

[20]D. Andrijivic Semi, pre-open sets, Mat, 1986, Vesnic, pp. 24-32.

[21]J. Weidmann, Spectral Theory of Ordinary Differential Operators, Springer-Verlag Berlin Heidelberg New York, 1987.

[22] P. Bhattacharya, B.K. Lahiri, Semi-generalized closed sets in topology, Indian J. Math. 29 (1987), 376382.

[23] N. Levine, Generalized closed sets in topology, Rand. Circ. Mat. Palermo, 19 (1970), 89- 96.

[24]Y. Gnanambal, On Generalized Pre-Regular Closed Sets in Topological Spaces, Indian J. Pure Appl. Math. 28(1997), 351-360. 